\title{
Antibiotic Resistance Profile and Distribution of Oxacillinase Genes Among Clinical Isolates of Acinetobacter baumannii in Shiraz Teaching Hospitals, 2012 - 2013
}

\author{
Sara Kooti ${ }^{1}$; Mohammad Motamedifar ${ }^{2,3,{ }^{*}}$; Jamal Sarvari ${ }^{2}$ \\ ${ }^{1}$ Student Research Committee, Bacteriology and Virology Department, School of Medicine, Shiraz University of Medical Sciences, Shiraz, IR Iran \\ ${ }^{2}$ Department of Bacteriology and Virology, School of Medicine, Shiraz University of Medical Sciences, Shiraz, IR Iran \\ ${ }^{3}$ Shiraz HIV|AIDS Research Center (SHARC), Shiraz University of Medical Sciences, Shiraz, IR Iran \\ ${ }^{*}$ Corresponding author: Mohammad Motamedifar, Department of Bacteriology and Virology, School of Medicine, Shiraz University of Medical Sciences, Shiraz, IR Iran. \\ Tel/Fax: +98-7132304356, E-mail: motamedm@sums.ac.ir
}

Received: May 14, 2014; Revised: June 16, 2014; Accepted: July 26, 2014

\begin{abstract}
Background: The emergence of multidrug-resistant Acinetobacter baumannii complicates the therapy of the related infections. Hospital isolates of A. baumannii are usually multidrug-resistant. The problem is compounded by increasing resistance to broad-spectrum antibiotics including carbapenems.

Objectives: The aim of this study was to determine antimicrobial susceptibility patterns and distribution of bla $O X A$-type carbapenemases genes among A. baumannii isolates from hospitalized patients in Shiraz, Southwest Iran.

Materials and Methods: Two hundred A. baumannii isolates were recovered from different clinical specimens in four Shiraz teaching hospitals. Isolates were detected as A. baumannii by Microgen kit and PCR with specific primers of bla OXA-51-like gene. Antimicrobial susceptibility testing was determined by disk diffusion method for all the isolates. Multiplex PCR assays were performed for detection of

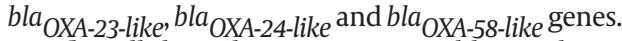

Results: All the isolates were susceptible to colistin and polymyxin B. Moreover, all of them were resistant to piperacillin, piperacillintazobactam, ampicillin, ceftazidime, cefoxitin and aztreonam. Eighty (40\%) isolates had positive results for bla $O X A-23-l i k e, 14$ (7\%) for bla $O X A$ 24-like and $1(0.5 \%)$ isolate for bla OXA-58-like. The co-existence of studied genes was detected for bla OXA-23-like $_{\text {plus }}$ bla OXA-24-like in nine $(4.5 \%)$ isolates.

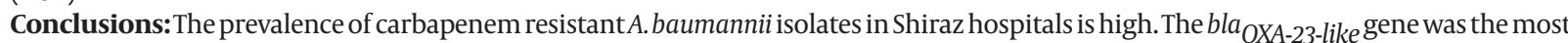
frequent carbapenemase identified among resistant $A$. baumannii isolated in Shiraz hospitals. The increasing incidence of $A$. baumannii is a serious concern, therefore control of this pathogen and taking preventive measures are emphasized.
\end{abstract}

Keywords: OXA-51 beta-lactamase; Oxacillinase; Acinetobacter baumannii

\section{Background}

Acinetobacter baumannii is a glucose non-fermentative Gram-negative bacillus classified as an opportunistic pathogen responsible for nosocomial infections, especially in Intensive Care Units (ICU) and Burn Therapy Units (BTU) $(1,2)$. A. baumannii is responsible for nosocomial pneumonia, wound infections, bacteremia, urinary tract infection, meningitis, endocarditis, osteomyelitis and keratitis (3). Also infections of respiratory tract, central nervous system, urinary tract and peritonitis can be caused by $A$. baumannii especially in immunocompromised patients (3). In the recent years, the emergence of multidrug-resistant $A$. baumannii has complicated the therapy of A. baumannii infections (4). Hospital strains of Acinetobacter are usually multidrug-resistant. MultidrugResistance (MDR) is defined as resistance to three or more representatives of the following classes of antibiotics: fluoroquinolones, third generation cephalosporins, aminoglycosides and carbapenems (5).
MDR is compounded by increasing resistance to broadspectrum antibiotics including carbapenems as one of the most effective antibiotics against Gram-negative rods $(6,7)$. Although, most of A. baumannii isolates were susceptible to carbapenems previously and imipenem was the most effective treatment for A. baumannii infections, widespread use of carbapenem has caused emergence of resistant strains $(8,9)$. The emergence of carbapenem resistance in A. baumannii is a significant public health concern (3). One of the major mechanisms of carbapenem resistance in this pathogen is production of carbapenem hydrolyzing beta- lactamases mostly Oxacillinase (OXA) types carbapenemases (10). Carbapenem resistance in A. baumannii is mediated by acquisition of a class B or class D beta-lactamase genes, such as oxacillinase genes (11). OXA-type carbapenamases have been divided into eight subgroups, four of which have been identified in $A$. baumannii: OXA-23-like, OXA-24-like, OXA-58-like and OXA-

Copyright (C) 2015, Ahvaz Jundishapur University of Medical Sciences. This is an open-access article distributed under the terms of the Creative Commons Attribution-NonCommercial 4.0 International License (http://creativecommons.org/licenses/by-nc/4.0/) which permits copy and redistribute the material just in noncommercial usages, provided the original work is properly cited. 
51-like (12). Recently, it has been suggested that enzymes belonging to the OXA-51-like subgroup are intrinsic to A. baumannii, occurring in most or all strains, although they are very variably expressed (13).

To control and prevent dissemination of resistant isolates, molecular method for typing of MDR A. baumannii is essential (14). The significant contribution of oxacillinase genes to carbapenem resistance has been emphasized, particularly when they are accompanied by ISAba1 and ISAba3 in the naturally occurring plasmids $(2,15)$. Several studies on these genes have been performed in the world and in some parts of Iran; however, there is no data on the distribution of bla OXA-type genes in A. baumannii isolates in Shiraz. Therefore, this study was performed to provide information in this regard. This study can help us to know the prevalence of bla OXA-type genes in A. baumannii isolates in our hospital settings for better infection control and treatment in hospitals in Shiraz.

\section{Objectives}

The aim of this study was to determine antimicrobial susceptibility patterns and distribution of bla OXA-type carbapenemase genes among $A$. baumannii isolates from hospitalized patients in four teaching hospitals, in Shiraz, Southwest Iran.

\section{Materials and Methods}

\subsection{Bacterial Strains}

From December 2013 to May 2013, 200 A. baumannii were isolated from different clinical specimens, including urine, wound, blood, sputum, endotracheal tube (ETT), body fluid, nose, throat and eye from four Shiraz teaching hospitals (Faghihi, Aliasghar, Ghotebedin and Nemazee) in Shiraz, southwest Iran. Some relevant information including sex, sample type and ward name was recorded. The study was approved by the Ethics Committee of Shiraz University of Medical Sciences. The specimens were cultured on MacConkey agar (Merck, Germany) and blood agar (Merck, Germany), then incubated at $37^{\circ} \mathrm{C}$ for 24 - 48 hours. These isolates were identified using standard biochemical tests, including Oxidase test, TSI (Triple Sugar Iron agar) medium (Merck, Germany) and SIM (Sulfide, Indole, Motility) medium (Merck, Germany). Negative result for oxidase test, no motility and non-fermentation and growth in temperature of $42-44^{\circ} \mathrm{C}$ was considered as the elementary criteria for A. baumannii detection. They were confirmed by Microgen kits (Mast, UK) according to the manufacturer instructions (www. microgenbioproducts.com).

\subsection{Antimicrobial Susceptibility Testing}

Antimicrobial susceptibility testing was performed by disk diffusion method according to the Clinical and Lab- oratory Standards Institute (CLSI) $(4,16)$. Disk diffusion method was performed on Muller-Hinton agar (Merck, Germany), using an inoculum of $10^{5} \mathrm{CFU}$. Antibiotic disks (Mast, UK) containing ampicillin $(10 \mu \mathrm{g})$, ampicillin-sulbactam $(10 \mu \mathrm{g})$, piperacillin $(100 \mu \mathrm{g})$, piperacillin-tazobactam $(110 \mu \mathrm{g})$, amikacin $(30 \mu \mathrm{g})$, gentamicin $(120 \mu \mathrm{g})$, imipenem $(10 \mu \mathrm{g})$, meropenem $(10 \mu \mathrm{g})$, ciprofloxacin (5 $\mu \mathrm{g})$, levofloxacin $(5 \mu \mathrm{g})$, ceftazidime $(30 \mu \mathrm{g})$, cefoxitine (30 $\mu \mathrm{g})$, aztreonam $(30 \mu \mathrm{g})$, colistin $(25 \mu \mathrm{g})$, polymyxin B (300 unit) and tigecycline $(15 \mu \mathrm{g})$. These antibiotic disks were then placed on agar plates and incubated at $37^{\circ} \mathrm{C}$ for 24 hours. Escherichia coli ATCC 25922 and Pseudomonas aeruginosa ATCC 27853 were used as quality controls strain in each susceptibility determination (6).

\subsection{DNA Extraction}

2 - 3 grown bacterial colonies on Muller-Hinton agar plates were suspended in $180 \mu \mathrm{L}$ PBS. Then DNA of the bacteria was extracted by phenol-chloroform (CinnaGen, Iran) method with some modifications as described before (14). They were used as template in Polymerase Chain Reaction (PCR) assay to amplify bla OXA carbapenemase genes (17).

\subsection{Detection of blaOXA-51-like Gene}

Identification of A. baumannii was confirmed using

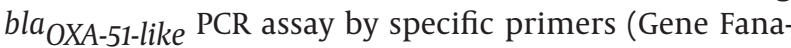
varan, Iran) (18). To amplify the bla $O X A$-51-like gene, each reaction final volume of $25 \mu \mathrm{L}$ was considered, containing 1x PCR buffer (CinnaGen, Iran), 1 U Taq polymerase (CinnaGen, Iran), $1.5 \mathrm{mM} \mathrm{Mgcl}_{2}$ (CinnaGen, Iran), $200 \mu \mathrm{M}$ of dNTP(CinnaGen, Iran), $10 \mathrm{pmol}$ of each primer and $1 \mu \mathrm{L}$ of extracted DNA. PCR conditions were programed in an Eppendorf Mastercycler as follows: Initial denaturation at $94^{\circ} \mathrm{C}$ for $3 \mathrm{~min}, 35$ cycles of $94^{\circ} \mathrm{C}$ for $45 \mathrm{~s}, 57^{\circ} \mathrm{C}$ for $45 \mathrm{~s}, 72^{\circ} \mathrm{C}$ for $1 \mathrm{~min}$ and 5 minute final extension of $72^{\circ} \mathrm{C}$. PCR products were electrophoresed (Bio Rad, USA) on $1.2 \%$ agarose gel (CinnaGen, Iran), stained with ethidium bromide solution $(1 \mathrm{mg} / \mathrm{mL})$ (CinnaGen, Iran) and placed under UV gel transilluminator. A. baumannii reference strain ATCC 19606 was used as positive control for bla OXA-51-like. Negative control was also included in each PCR reaction, containing all components except the DNA template which was replaced by distilled water $(6,14)$.

\subsection{Multiplex PCR Assay for Detection of Oxacillin- ase Genes}

A Multiplex PCR targeting bla OXA-23-like, bla OXA-24-like and bla $O X A$-58-like genes was used to screen the isolates as previously described (17). All the isolates were subjected to Multiplex PCR performed to detect bla $O X A-23$-like, bla $_{\text {OXA-24-like and bla }}$ OXA-58-like using specific primers (Gene Fanavaran, Iran), as listed in Table 1 (19). Final volume of $25 \mu \mathrm{L}$ included 1x PCR buffer, $1 \mathrm{U}$ Taq polymerase, $2 \mathrm{mM} \mathrm{MgCl}_{2}, 200 \mu \mathrm{M}$ of dNTP, $0.2 \mu \mathrm{M}$ of each primer and 
Kooti S et al.

$1 \mu \mathrm{L}$ of template DNA. Amplification protocol was initial denaturation at $94^{\circ} \mathrm{C}$ for $5 \mathrm{~min}, 94^{\circ} \mathrm{C}$ for $30 \mathrm{~s}, 53^{\circ} \mathrm{C}$ for $40 \mathrm{~s}$ and $72^{\circ} \mathrm{C}$ for $50 \mathrm{~s}$ and final extension at $72^{\circ} \mathrm{C}$ for $6 \mathrm{~min}$. The PCR products were run in $2.5 \%$ agarose gel electrophoresis, stained with ethidium bromide (CinnaGene, Iran) and visualized under UV gel transilluminator. A. baumannii reference strains NCTC 13304, NCTC 13302 and NCTC 13305 were used as positive control for bla $O X A-23-l i k e$, blaOXA-24-like and bla OXA-58-like, respectively $(6,14)$.

\subsection{Statistical Analyses}

To determine the association between variables, data was analyzed by Chi-square and Fisher's exact test using SPSS, version 21 (SPSS Inc. Chicago, IL, USA). P values $<0.05$ were considered as statistically significant.

\section{Results}

\subsection{Bacterial Isolates}

Of two hundred isolates, 109 (54.5\%) were from male and 91 (45.5\%) from female patients. Isolates were collected from four hospitals; 82 (41\%) from Nemazee hospital, 53 (26.5\%) from Aliasghar hospital, 48 (24\%) from Faghihi hospital and 17 (8.5\%) from Ghotbedin Hospital. The rates of isolation from different wards were as follows; ICU 143 (71.5\%), surgeries ward 16 (8\%), neurosurgical ICU 14 (7\%), neonates 8 (4\%), female internal ward 7 (3.5\%), male internal ward 7 (3.5\%) and organ transplantation ward 5 (2.5\%). Of two hundred isolates, 81 (40.5\%) were from sputum, 43 (21.5\%) from endotracheal tube, 22 (11\%) from wound, 16 (8\%) from urine, 12 (6\%) from blood, 12 (6\%) from body fluids, 4 (2\%) from nose, 3 (1.5\%) from throat, 2 (1\%) from CSF, $1(0.5 \%)$ from eye specimens and $4(2 \%)$ other samples. All A. baumannii isolates $(\mathrm{n}=200)$ in this study had positive results for bla $O X A-51$-like by PCR (Figure 1).

\subsection{Antimicrobial Susceptibility Testing}

All the isolates were susceptible to colistin and polymyxin B and all were MDR as defined before, they were totally resistant to piperacillin, piperacillin-tazobactam, ampicillin, ceftazidime, cefoxitin and aztreonam (Table 2). The association between source of isolates and antibiotic resistance pattern was not statistically significant $(\mathrm{P}>0.05)$.

\subsection{Detection of Oxacillinase Genes}

All isolates of A. baumannii were tested by Multiplex PCR for the presence of oxacillinase genes. Eighty (40\%) of 200 isolates had positive results for bla OXA-23-like, 14 (7\%) for bla $O X A-24$-like and 1 (0.5\%) isolate for bla $O X A$ 58-like. The co-existence of two different bla $O X A$ genes in the samples was detected for bla $O X A$-23-like plus bla $O X A$ 24-like in nine (4.5\%) isolates (Figure 2 ). The association between source of isolates and presence of genes was not statistically significant.
Table 1. Primer Sequences Used in This Study to Detect Oxacillinase Genes

\begin{tabular}{lc}
\hline Primer Sequence (5' $\left.\mathbf{~} \mathbf{3}^{\prime}\right)$ & Amplicon Size, bp \\
\hline blaOXA-23-like & 501 \\
\hline GATCGGATTGGAGAACCAGA & \\
\hline ATTTCTGACCGCATTTCCAT & 246 \\
\hline blaOXA-24-like & \\
\hline GGTTAGTTGGCCCCCTAAAA & 353 \\
\hline AGTTGAGCGAAAAGGGGATT \\
\hline blaOXA-51-like \\
\hline TAATGCTTTGATCGGCCTTG \\
\hline TGGATTGCACTTCATCTTGG \\
\hline blaOXA-58-like \\
\hline AAGTATTGGGGCTTGTGCTG \\
\hline CCCCTCTGCGCTCTACATAC \\
\hline
\end{tabular}

Figure 1. Agarose Gel Electrophoresis of bla OXA-51-like Gene in Acinetobacter baumannii

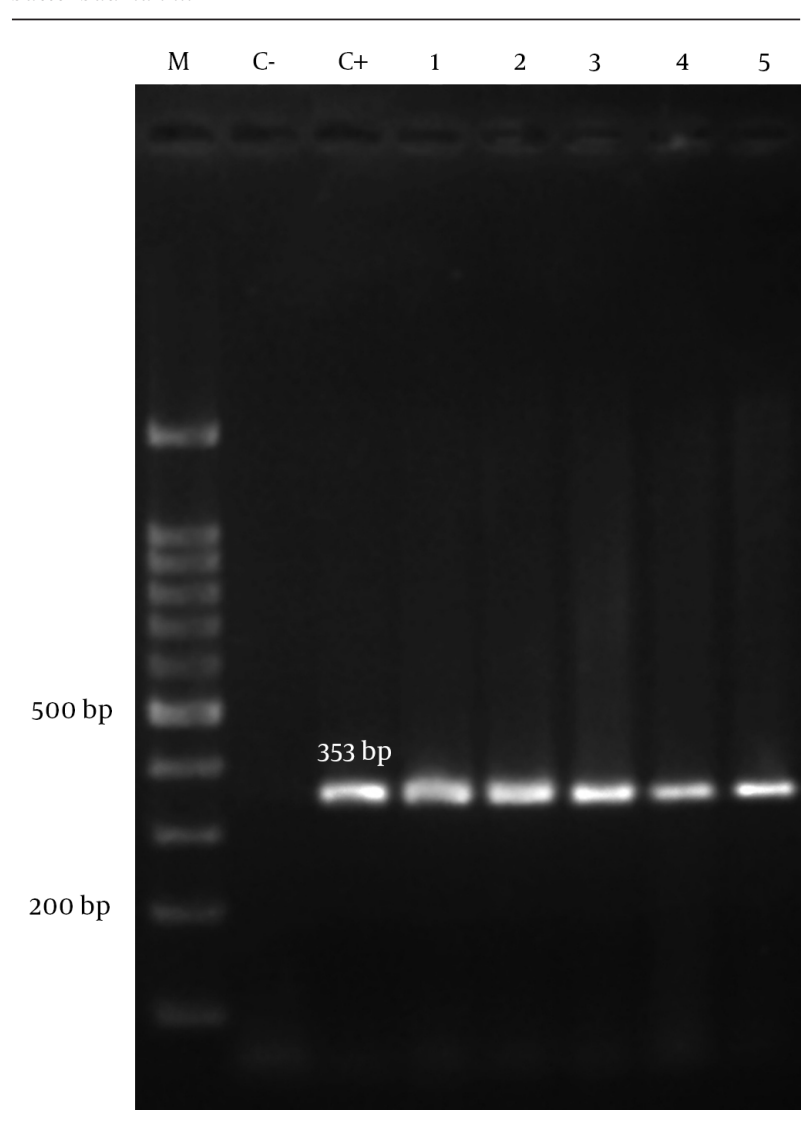

1, 2, 3, 4, 5, clinical isolates of A. baumannii with bla $O X A-51$-like gene; C-, negative control; $\mathrm{C}+$, positive control; $\mathrm{M}, 100 \mathrm{bp}$ DNA ladder. 
Kooti S et al.

\begin{tabular}{lccc}
\hline Table 2. The Results of Antibiogram for Studied A. baumannii Isolates ${ }^{\text {a }}$ & & \\
\hline Antibiotics & Sensitive & Intermediate & Resistant \\
\hline Imipenem & $2(1)$ & $1(0.5)$ & $197(98.5)$ \\
\hline Meropenem & $1(0.5)$ & - & $199(99.5)$ \\
Piperacillin & - & - & $200(100)$ \\
Piperacillin-tazobactam & - & - & $200(100)$ \\
Ampicillin & - & - & $200(100)$ \\
Ampicillin-sulbactam & $6(3)$ & $3(1.5)$ & $191(95.5)$ \\
Ceftazidime & - & $1(0.5)$ & $199(99.5)$ \\
Cefoxitine & - & - & $200(100)$ \\
Gentamicin & $27(13.5)$ & $4(2)$ & $169(84.5)$ \\
Amikacin & $17(8.5)$ & $10(5)$ & $173(86.5)$ \\
Ciprofloxacin & $1(0.5)$ & - & $199(99.5)$ \\
\hline Levofloxacin & $1(0.5)$ & - & $199(99.5)$ \\
\hline Aztreonam & - & - & $200(100)$ \\
Colistin & $200(100)$ & - & - \\
\hline Polymyxin B & $200(100)$ & - & - \\
\hline Tigecycline & $175(87.5)$ & $21(10.5)$ & $4(2)$ \\
\hline Val
\end{tabular}

${ }^{\mathrm{a}}$ Values are presented as No(\%).

Figure 2. Agarose Gel Electrophoresis of bla OXA-type Carbapenemases by Multiplex PCR

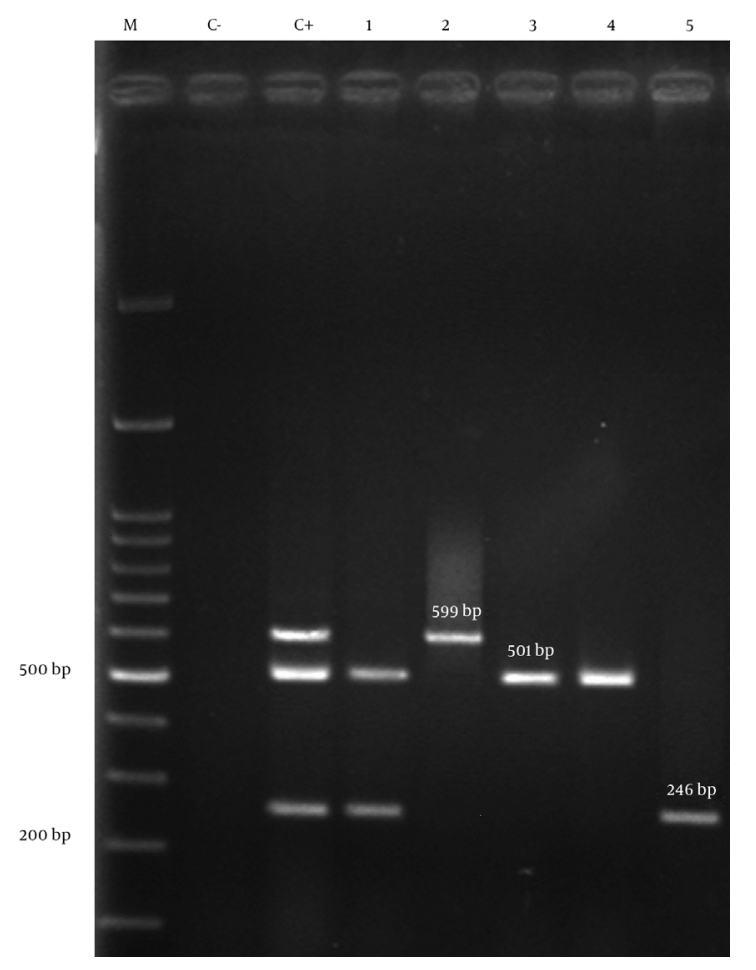

1, clinical isolate of A. baumannii containing bla ${ }_{O X A-23-l i k e}$ and bla ${ }_{O X A}$ 24-like genes; 2, clinical isolate of A. baumannii having bla OXA-58-like gene 3,4, clinical isolates of A. baumannii having bla $O X$-23-like genes; 5, clinical isolate of A. baumannii with bla $O X A-24-l i k e$ gene; C-, negative control; $\mathrm{C}+$, positive control (A. baumannii NCTC 13304, NCTC 13302, NCTC 13305 for bla ${ }_{\text {OXA-23-like, }}$ bla OXA-24-like, bla $_{\text {OXA-58-like }}$ ); M,100 bp DNA ladder.

\section{Discussion}

Acinetobacter baumannii is an emerging nosocomial pathogen which is in part due to its capacity of acquiring resistance to multiple antimicrobial agents (20). Occurrence of multidrug-resistance and Pandrug-Resistant (PDR) in A. baumannii is a growing concern (7). All of the isolates in this study were multidrug-resistant and resistant to most of the antibiotics (Table 2). This pathogen is an important Gram-negative bacterium involved in nosocomial infections, especially in ICU wards (18). As shown in our study, $71.5 \%$ of the isolates were obtained from hospitalized patients in ICU. This result confirms the fact that A. baumannii is often an important cause of infection in hospitalized patients in ICU.

To detect antibiotic resistant patterns, the isolates were investigated primarily by phenotypic methods (21). Our results revealed that $98.5 \%$ and $99.5 \%$ of the isolates were resistant to imipenem and meropenem, respectively. In some studies in Tehran, 50.9\%, 52.5\%, 62\% and $67.5 \%$ of the isolates were resistant to imipenem and 51.8\%, 52.5\%, $62 \%$ and $84.5 \%$ to meropenem in 2008, 2009, 2011 and 2013, respectively $(6,12,18,20)$. In another study in Tehran in 2013 , resistance rates of $99 \%$ and $91.5 \%$ were reported to imipenem and meropenem, respectively (22). Mirnejad and Vafaei, reported $76 \%$ resistance to imipenem and 69\% resistance to meropenem among tested isolates in Tehran in 2013 (23). In two separate studies, one in Ahvaz in 2013, 96.1\% resistance to imipenem and meropenem (14), and another in Kermanshah in 2013, 79.8\% resistance to imipenem and $75 \%$ resistance to meropenem were reported (15). Therefore, our results showed that resistance to carbapenems has an increasing trend which is proba- 
bly because of dissemination of highly resistant lineages of $A$. baumannii in our area. Increased resistance to carbapenem causes a real concern over an imminent threat of untreatable A. baumannii infections (3).

The current study showed low susceptibility rates to most of available antimicrobial agents for the treatment of infections caused by A. baumannii, except for polymyxin B and colistin. In our study, all of the isolates were sensitive to colistin and polymyxin B, while other studies conducted in Tehran demonstrated $12 \%$ resistance to colistin and 3\% resistance to polymyxin B in 2011 (17). Moreover, in two studies in Tehran and Ahvaz in 2013, all of the isolates were sensitive to these antibiotics $(12,14)$. In a study in Kermanshah in 2013, all isolates were sensitive to polymyxin B and colistin (15). Sensitivity of all $A$. baumannii isolates to colistin was also reported in Tehran in 2013 (22). Also in a study in Tehran in 2013, among all antibiotic tested, the lowest resistance rate to polymyxin B (3\%) was observed (23). The current study also described the important role of class D carbapenem hydrolyzing beta-lactamases. Production of class $\mathrm{D}$ oxacillinase by A. baumannii distributed worldwide is the main mechanism of resistance to carbapenems in this organism. The major carbapenemase genes involved in carbapenem resistance in A. baumannii are bla OXA-23-like, bla OXA-24-like $_{\text {OXA-23-like }}$ and bla $O X A-58$-like $(8,14)$. Alleles encoding OXA-23-like, OXA-24-like and OXA-58-like enzymes were consistently associated with resistance or at least reduced susceptibility to carbapenemases (24).

Our PCR results revealed that all A. baumannii isolates had bla $O X A$-51-like gene. The bla $O X A$-51-like genes are located intrinsically in chromosome of all A. baumannii strains (8). This result provides evidence that detection of bla OXA-51-like can be used as a simple and reliable way of identifying A. baumannii (13). GenBank submissions describing variants from isolates of $A$. baumannii from many different countries distributed over four continents clearly suggest that bla OXA-51-like is ubiquitous in A. baumannii (25). Distribution of other bla OXA-type genes in A. baumannii is variable. OXA-23-like was the first OXAtype beta-lactamase identified in A. baumannii (26). The results obtained in this study indicated that most of our isolates (40\%) carried the bla OXA-23-like gene. In other studies investigating outbreaks of OXA-23-producing $A$. baumannii strains, the rate of bla OXA-23-like ranged 31\% to $94 \%$ in different parts of the world (14). So our results for bla OXA-23-like gene are in the reported ranges. In both separate studies in Tehran, in 2008 and 2009, 25\% of the isolates had positive results for bla OXA-23-like gene (6, 20). Moreover, in other two studies by Karmostaji et al. (12) and Goudarzi et al. (22), in Tehran in 2013, 81.3\% and $55.7 \%$ had positive findings for this gene, respectively. $85 \%$ and $55.7 \%$ positivity rates were reported for bla OXA-23-like gene in studied A. baumannii isolates in Ahvaz and Kermanshah, respectively $(14,15)$.

In our study, $7 \%$ of the $A$. baumannii isolates had positive results for bla OXA-24-like gene. The reported fre- quency rate of this gene has been previously reported as $0-85.4 \%$ in different parts of the world (14). In a study in Tehran in $2008,17.9 \%$ of isolates contained bla OXA24-like gene (6); In addition, in another study in Tehran in $2009,15 \%$ had positive results for this gene (20). Three studies in 2013 in Tehran, Ahvaz and Kermanshah showed frequencies of $8.13 \%, 8.7 \%$ and $19.2 \%$ for this gene in the studied isolates, respectively $(12,14,15)$. Some authors reported bla $O X A-58$-like frequency as $2-84.9 \%$ in $A$. baumannii isolates in different parts of the world (14). In several studies in Tehran in 2008, 2009 and 2013, $9 \%, 21.2 \%$ and $0.8 \%$ of the isolates had positive results for bla OXA-58-like, respectively $(6,12,20)$. Moreover, a study conducted in Ahvaz and Kermanshah, in 2013, revealed that bla OXA-58-like gene was not detected $(14,15)$. In our study, we identified bla OXA-58-like gene only in one $A$. baumannii isolate $(0.5 \%)$. This frequency was similar to the rate previously reported from central Iran.

We identified nine isolates (4.5\%) with co-existence of two different bla OXA-23-like plus bla OXA-24-like. In two studies in 2013, in Tehran and Kermanshah, 5.7\% and $16.4 \%$ of A. baumannii isolates had such a co-existence (12, 15). In another study in 2013 in Ahvaz, no co-existence between these genes was reported (14). Of note, $105 \mathrm{~A}$. baumannii isolates resistant to imipenem and meropenem in our study possessed only the intrinsic bla OXA-51-like, but they had negative result for other investigated genes. Resistance to carbapenems in those isolates may be due to other mechanisms other than oxacillinase production $(14,27)$.

In conclusion, our study confirmed that MDR A. baumannii strains were disseminated in Shiraz, Iran. Such $A$. baumannii with different bla ${ }_{O X}$-carbapenemase genes were isolated from hospitalized patients at Shiraz teaching hospitals (Nemazee, Faghihi, Aliasghar, Ghotbedin) in different wards. The bla OXA-51-like genes were the most prevalent subgroup, as they are intrinsic to A. baumannii. Moreover, bla OXA-23-like gene is another most prevalent resistance gene among MDR A. baumannii isolates. The distribution of bla OXA-58-like gene was low in our study. Controlling infections of MDR A. baumannii in hospitals needs a common strategy issued by decision makers and health-care authorities to make hospitals a safer place for patients.

\section{Acknowledgements}

The authors would like to express their sincere appreciation to Dr. Saeed Shoja from Ahvaz Jundishapur University of Medical Sciences for his provision of positive controls for PCR.

\section{Authors' Contributions}

Study idea, design and the protocol: Mohammad Motamedifar and Sara Kooti; sample collection, experiments, possession of raw data: Sara Kooti; data analysis, critical revision of the manuscript for important intellectual 
content: Mohammad Motamedifar and Jamal Sarvari; final approval of the entire study: Mohammad Motamedifar and Jamal Sarvari. Sara Kooti, and Mohammad Motamedifar wrote the manuscript.

\section{Funding/Support}

This study was financially supported by Shiraz University of Medical Sciences, grant No.92-6488. This paper was extracted from MSc thesis of Sara Kooti, supervised by Mohammad Motamedifar.

\section{References}

1. Opazo A, Dominguez M, Bello H, Amyes SG, Gonzalez-Rocha G. OXA-type carbapenemases in Acinetobacter baumannii in South America. J Infect Dev Ctries. 2012;6(4):311-6.

2. Peleg AY, Seifert H, Paterson DL. Acinetobacter baumannii: emergence of a successful pathogen. Clin Microbiol Rev. 2008;21(3):538-82.

3. Maragakis LL, Perl TM. Acinetobacter baumannii: epidemiology, antimicrobial resistance, and treatment options. Clin Infect Dis. 2008;46(8):1254-63.

4. Peymani A, Higgins PG, Nahaei MR, Farajnia S, Seifert H. Characterisation and clonal dissemination of OXA-23-producing Acinetobacter baumannii in Tabriz, northwest Iran. Int J Antimicrob Agents. 2012;39(6):526-8.

5. Magiorakos AP, Srinivasan A, Carey RB, Carmeli Y, Falagas ME, Giske CG, et al. Multidrug-resistant, extensively drug-resistant and pandrug-resistant bacteria: an international expert proposal for interim standard definitions for acquired resistance. Clin Microbiol Infect. 2012;18(3):268-81.

6. Feizabadi MM, Fathollahzadeh B, Taherikalani M, Rasoolinejad M, Sadeghifard N, Aligholi M, et al. Antimicrobial susceptibility patterns and distribution of blaOXA genes among Acinetobacter spp. Isolated from patients at Tehran hospitals. Jpn J Infect Dis. 2008;61(4):274-8.

7. Gaynes R, Edwards JR, National Nosocomial Infections Surveillance $S$. Overview of nosocomial infections caused by gram-negative bacilli. Clin Infect Dis. 2005;41(6):848-54

8. Kempf M, Rolain JM. Emergence of resistance to carbapenems in Acinetobacter baumannii in Europe: clinical impact and therapeutic options. Int J Antimicrob Agents. 2012;39(2):105-14.

9. Shete VB, Ghadage DP, Muley VA, Bhore AV. Multi-drug resistant Acinetobacter ventilator-associated pneumonia. Lung India. 2010;27(4):217-20.

10. Irfan S, Turton JF, Mehraj J, Siddiqui SZ, Haider S, Zafar A, et al. Molecular and epidemiological characterisation of clinical isolates of carbapenem-resistant Acinetobacter baumannii from public and private sector intensive care units in Karachi, Pakistan. $J$ Hosp Infect. 2011;78(2):143-8.

11. Lin MF, Chang KC, Lan CY, Chou J, Kuo JW, Chang CK, et al. Molecular epidemiology and antimicrobial resistance determinants of multidrug-resistant Acinetobacter baumannii in five proximal hospitals in Taiwan. Jpn J Infect Dis. 2011;64(3):222-7.

12. Karmostaji A, Najar Peerayeh S, Hatef Salmanian A. Distribution of OXA-Type Class D $\beta$-Lactamase Genes Among Nosocomial
Multi Drug Resistant Acinetobacter baumannii Isolated in Tehran Hospitals. Jundishapur J Microbiol. 2013;6(5):1-5.

13. Turton JF, Woodford N, Glover J, Yarde S, Kaufmann ME, Pitt TL. Identification of Acinetobacter baumannii by detection of the blaOXA-51-like carbapenemase gene intrinsic to this species. $J$ Clin Microbiol. 2006;44(8):2974-6.

14. Shoja S, Moosavian M, Peymani A, Tabatabaiefar MA, Rostami S Ebrahimi N. Genotyping of carbapenem resistant Acinetobacter baumannii isolated from tracheal tube discharge of hospitalized patients in intensive care units, Ahvaz, Iran. Iran J Microbiol. 2013;5(4):315-22.

15. Mohajeri P, Farahani A, Feizabadi MM, Ketabi H, Abiri R, Najafi F. Antimicrobial susceptibility profiling and genomic diversity of Acinetobacter baumannii isolates: A study in western Iran. Iran J Microbiol. 2013;5(3):195-202.

16. Clinical and Laboratory Standards Institude (CLSI).. Performance standards for antimicrobial susceptibility testing. $12 \mathrm{ed}$ : Clinical and Laboratory Standards Institude (CLSI); 2010.

17. Shahcheraghi F, Abbasalipour M, Feizabadi M, Ebrahimipour G, Akbari N. Isolation and genetic characterization of metallobeta-lactamase and carbapenamase producing strains of Acinetobacter baumannii from patients at Tehran hospitals. Iran $J$ Microbiol. 2011;3(2):68-74.

18. Sohrabi N, Farajnia S, Akhi MT, Nahaei MR, Naghili B, Peymani A, et al. Prevalence of OXA-type beta-lactamases among Acinetobacter baumannii isolates from Northwest of Iran. Microb Drug Resist. 2012;18(4):385-9.

19. Poirel L, Walsh TR, Cuvillier V, Nordmann P. Multiplex PCR for detection of acquired carbapenemase genes. Diagn Microbiol Infect Dis. 2011;70(1):119-23.

20. Taherikalani M, Fatolahzadeh B, Emaneini M, Soroush S, Feizabadi MM. Distribution of different carbapenem resistant clones of Acinetobacter baumannii in Tehran hospitals. New Microbiol. 2009;32(3):265-71.

21. Nowak P, Paluchowska P, Budak A. Distribution of blaOXA genes among carbapenem-resistant Acinetobacter baumannii nosocomial strains in Poland. New Microbiol. 2012;35(3):317-25.

22. Goudarzi H, Douraghi M, Ghalavand Z, Goudarzi M. Assessment of antibiotic resistance pattern in Acinetobacter bumannii carrying bla oxA type genes isolated from hospitalized patients. Novel Biomed. 2013;1(2):54-61.

23. Mirnejad R, Vafaei S. Antibiotic resistance patterns and the prevalence of ESBLs among strains of Acinetobacter baumannii isolated from clinical specimens. The Journal of Genes, Microbes and Immunity. 2013;2013:1-8.

24. Woodford N, Ellington MJ, Coelho JM, Turton JF, Ward ME, Brown S, et al. Multiplex PCR for genes encoding prevalent OXA carbapenemases in Acinetobacter spp. Int J Antimicrob Agents. 2006;27(4):351-3.

25. Brown S, Amyes SG. The sequences of seven class D beta-lactamases isolated from carbapenem-resistant Acinetobacter baumannii from four continents. Clin Microbiol Infect. 2005;11(4):326-9.

26. Paton R, Miles RS, Hood J, Amyes SG, Miles RS, Amyes SG. ARI 1 beta-lactamase-mediated imipenem resistance in Acinetobacter baumannii. Int J Antimicrob Agents. 1993;2(2):81-7.

27. Kulah C, Mooij MJ, Comert F, Aktas E, Celebi G, Ozlu N, et al. Characterisation of carbapenem-resistant Acinetobacter baumanni outbreak strains producing OXA-58 in Turkey. Int J Antimicrob Agents. 2010;36(2):114-8. 